\title{
Effect of salinity on carbon and sulfur cycles in Umm Alhool sabkha microbial mat ecosystem in Qatar
}

\author{
Mohammad Al-Najjar, Roda Al-Thani, Abdul Monem Al Raei
}

King Abdullah University of Science and Technology, Thuwal, SAUDI ARABIA; Dept. of Biological and Environmental Sciences, Doha, QATAR; Max Planck Institute for Marine Microbiology, Bremen, GERMANY

malnajja@mpi-bremen.de

Microbial mats are only present under extreme conditions, where grazing by higher organisms is limited. Therefore, microbial mats may provide insight into extraterrestrial life, due to their adaptations to extreme temperatures, desiccation or salinity. They are faced with a diurnal cycle with variable length based on their location, which exposes them to extreme salinity conditions (i.e., water withdrawal and high evaporation). Cyanobacteria in the photic zone of a mat ecosystem supply the other microorganism with the required organic material to produce energy and grow. Subsequently, this will reproduce the nutrients needed by the phototrophs through elemental re-mineralization.

In this work, we investigated the effect of water salinity that covers the microbial mat ecosystem of Umm Alhool sabkha, Qatar, regarding the most important processes within microbial mats: photosynthesis and sulfate reduction (SR). Our results showed that both photosynthetic and sulfate reduction rates decreased with increasing the salinity. The microbial community structure, assessed by 454 pyro-sequencing, revealed that the cyanobacterial community structure changed in response to the change in salinity. This was not the case for the sulfate reducer community structure, which stayed as it is in the mats incubated at different salinities. Therefore, we speculate that salinity affects the photosynthetic community structure, and consequently affects the photosynthetic activity of the whole ecosystem. However, sulfate reduction rates decreased due to less organic material supply from the upper layers and not due to change in microbial community structure of SR. Other factors such as the activity of the enzymes could also have an effect on SRR, but it was not investigated in this study. 